\title{
Estudio de indicadores de suelo y planta para la programación del riego en cerezos jóvenes
}

\author{
Blaya-Ros, P.J.'; Blanco, V. ${ }^{1}$, Torres-Sánchez, R. ${ }^{1}$, González-Teruel, J.D. ${ }^{1}$, Espósito, M.E. ${ }^{2}$, Domingo, R. ${ }^{1}$
}

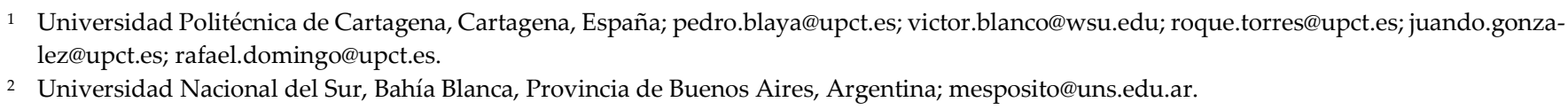

2 Universidad Nacional del Sur, Bahía Blanca, Provincia de Buenos Aires, Argentina; mesposito@uns.edu.ar.

Resumen: En zonas áridas y semiáridas existe una necesidad constante de mejorar la eficiencia del uso del agua. En este sentido, el empleo de indicadores puede convertirse en una herramienta eficaz para confeccionar programas de riego más precisos. Durante el verano de 2018 y 2019, con el objetivo de estudiar la sensibilidad y la variabilidad de distintos indicadores de suelo y planta, se llevó a cabo un ensayo en cerezos jóvenes (Prunus avium L. 'Lapins'). Tres tratamientos de riego aplicados fueron: control (CTL) regado para asegurar condiciones no limitantes de agua en el suelo, y dos tratamientos deficitarios, MS y SS, los cuales fueron regados como CTL hasta finales de junio, momento a partir del cual se alternaron 2 ciclos consecutivos de supresión - reanudación del riego. Los árboles fueron recupera-

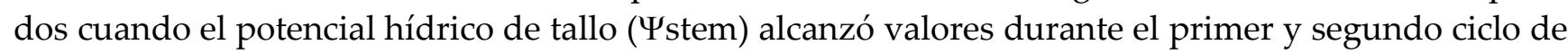
supresión de -1,3 y -1,7 MPa para MS y -1,6 y -2,3 MPa para SS. Los indicadores de suelo y planta estudiados fueron afectados por el déficit hídrico impuesto. La tasa de crecimiento diaria del tronco (TGR) y la máxima contracción diaria del tronco (MDS) mostraron una respuesta rápida al cambio de riego; sin embargo, ambos indicadores tuvieron una alta variabilidad. En este sentido, MDS mostró la mayor intensidad de señal, aunque la sensibilidad de este indicador fue mitigada por su coeficiente de variación $(\mathrm{CV})$. Por otro lado, el contenido volumétrico de agua del suelo $(\theta \mathrm{v})$ y la temperatura de la copa (Tc) exhibieron una alta sensibilidad debido a la baja variabilidad. $\Psi$ stem, indicador usado como referencia, mostró una estrecha relación con el resto de los indicadores estudiados, excepto con TGR. La relación observada entre MDS y $\Psi$ stem fue lineal hasta alcanzar valores de $\Psi$ stem de -1,3 MPa. Los resultados obtenidos sugieren que $\Psi$ stem es un indicador sensible al estrés con un bajo CV; Tc, la diferencia de temperatura de la copa y del aire y el índice de estrés del cultivo pueden ayudar a determinar, de forma rápida y sencilla, el estado hídrico del cultivo; y MDS podrías ser utilizado hasta valores de estrés moderados para la elaboración de programas de riego en cerezos jóvenes.

Palabras clave: Programación del riego, indicadores de estrés hídrico; variabilidad; sensibilidad; Prunus avium L.; Potencial hídrico de tallo; Temperatura de la copa. 


\title{
Feasibility of soil and plant indicators for irrigation scheduling in cherry trees
}

\author{
Blaya-Ros, P.J. ${ }^{1}$; Blanco, V. ${ }^{1}$, Torres-Sánchez, R. ${ }^{1}$, González-Teruel, J.D. ${ }^{1}$, Espósito, M.E. ${ }^{2}$, Domingo, R. ${ }^{1}$ \\ Universidad Politécnica de Cartagena, Cartagena, España; pedro.blaya@upct.es; victor.blanco@wsu.edu; roque.torres@upct.es; juando.gonza- \\ lez@upct.es; rafael.domingo@upct.es. \\ 2 Universidad Nacional del Sur, Bahía Blanca, Provincia de Buenos Aires, Argentina; mesposito@uns.edu.ar.
}

\begin{abstract}
In arid and semi-arid areas there is a constant need to improve water use efficiency. In this sense, the use of indicators can become an effective tool to design more accurate irrigation programs. During the summer of 2018 and 2019, with the aim of studying the sensibility and variability of different soil and plant indicators, an experiment was carried out in young 'Lapins' cherry trees (Prunus avium L.). Three irrigation treatments were applied: control treatment (CTL) irrigated to ensure non-limiting soil water conditions; moderate water stress (MS) subjected to two drying cycles whose duration was dependent on the time elapsed until the trees reached values of midday stem water potential (Ystem) of -1.3 and $-1.7 \mathrm{MPa}$ for the first and second cycle, respectively; and severe water stress (SS) similar to MS, but with reference values of -1.6 and $-2.5 \mathrm{MPa}$. In-between drought cycles, the MS and SS trees were irrigated daily as the CTL trees until reaching $\Psi$ stem values like the CTL trees. The soil and plant indicators studied were affected by the water deficit. Trunk growth rate (TGR) and maximum daily shrinkage (MDS) showed a rapid response to irrigation alteration; however, both indicators had high variability. In this sense, MDS showed the highest signal intensity, although its sensitivity was reduced by its coefficient variability $(\mathrm{CV})$. On the other hand, the volumetric water content $(\theta \mathrm{v})$ and the canopy temperature (Tc) showed a high sensitivity due to low variability. $\Psi$ stem, the indicator used as a reference, showed a close relationship with other indicators, except for TGR. The relationship obtained between MDS and $\Psi$ stem was linear until reaching values of $\Psi$ stem of -1.3 MPa. These results obtained suggest that $\Psi$ stem is a stress-sensitive indicator with a low $\mathrm{CV}$; Tc, the difference between the canopy and the air and crop water stress index can help to determine, quickly and easily, the crop water status; and MDS could be used up to moderate stress values for deficit irrigation scheduling in young cherry trees.
\end{abstract}

Keywords: Irrigation scheduling, water stress indicators; variability; sensitivity; Prunus avium L.; stem water potential; canopy temperature. 


\section{Congreso Nacional de Riegos CARTAGENA 2021}

\section{Introducción}

En las zonas áridas y semiáridas, la frecuencia e intensidad de los periodos de sequía se han incrementado debido al cambio climático, lo que podría provocar una reducción de la disponibilidad hídrica. Esta situación ha generado la necesidad de optimizar el uso del agua en el sector agrícola en estas zonas, principal consumidor de los suministros hídricos. Sin embargo, para aumentar la eficiencia en el uso del agua es necesario conocer, de forma directa o indirecta, el estado hídrico de la planta.

Números indicadores del estado hídrico del suelo y de la planta han sido utilizados para la confección y supervisión de programas de riego. Los indicadores de suelo son ampliamente empleados a nivel comercial para la programación del riego, debido a que pueden determinar de forma indirecta el estado hídrico de la planta, además de ser fácilmente interpretables y automatizables [1]. Por el contrario, debido a su mayor dificultad de interpretación y medida, los indicadores del estado hídrico de la planta son aplicados principalmente en el ámbito de la investigación. El potencial hídrico de tallo y de hoja han sido los indicadores de referencia para estimar el estado hídrico de la planta [2]. Si bien, su aplicabilidad en campo es compleja, debido a que es una técnica muy laboriosa y no automatizable. Por esta razón, aumentó el interés en la utilización de las fluctuaciones de diámetro de tronco en la programación del riego en frutales. Los cambios en el diámetro del tronco se han utilizado con éxito en múltiples frutales [3,4], debido a que son capaces de monitorizar el estado hídrico del cultivo de forma continua. Por otro lado, la termografía infrarroja ha emergido como una herramienta no destructiva y capaz de estimar de forma precisa el estado hídrico de los cultivos a distintas escalas [5]. La temperatura de la copa (Tc) es regulada principalmente por la tasa transpirativa de la planta y, por lo tanto, por la apertura estomática.

Teniendo en cuenta lo anteriormente comentado, se llevó a cabo durante dos años un experimento en cerezos (Prunus avium L.) sometidos a varios ciclos de supresión-recuperación del riego con el objetivo de estudiar la sensibilidad, la variabilidad y la adecuación de distintos indicadores de suelo y planta para su aplicación en el manejo del riego.

\section{Materiales y métodos}

El estudio se llevó a cabo durante junio - septiembre de 2018 y 2019 en la Estación Experimental Agroalimentaria 'Tomás Ferro' (ETSIA-UPCT), ubicada en Cartagena. El material vegetal empleado fueron cerezos ( $P$. avium L. 'Lapins') injertados sobre 'Mirabolano'. Los árboles tenían 3 años de edad al inicio del ensayo y estaban dispuesto en un marco de plantación de 3,5 m x 2,25 m. El sistema de riego constó de un único lateral de riego por hilera de árboles con tres emisores autocompensantes de 2,2 $\mathrm{L} \mathrm{h}^{-1}$ por planta. El agua de riego presentó una conductividad eléctrica $\left(\mathrm{CE}_{25^{\circ} \mathrm{C}}\right)$ de $1,1 \mathrm{dS} \mathrm{m}^{-1} \mathrm{y}$ fue de buena calidad en general.

El diseño experimental fue de bloques al azar, con 3 repeticiones por tratamiento. Los tratamientos de riego fueron: i) CTL, control, regado para satisfacer el $115 \%$ de las necesidades hídricas del cultivo, ii) MS, déficit moderado-severo, regado como CTL hasta finales de junio, momento a partir del cual se alternaron 2 ciclos consecutivos de supresión-reanudación del riego. El riego se reanudó tras alcanzar un potencial hídrico de tallo ( $\Psi$ stem) de $-1,3 \mathrm{MPa}$ en el primer ciclo de sequía y de -1,6 MPa en el segundo; y iii) SS, déficit severo, similar a MS, pero con recuperaciones a -1,6 MPa y -2,3 MPa. Los periodos de recuperación se dieron por finalizados cuando los valores de $\Psi$ stem de los tratamientos deficitarios alcanzaron valores de $\Psi$ stem similares a los de los árboles CTL.

Las variables meteorológicas fueron proporcionadas por la estación agroclimática CA12 del Servicio de Información Agraria de la Región de Murcia (SIAM - https://siam.imida.es/). La dosis de riego fue calculada semanalmente a partir de la evapotranspiración del cultivo (ETc), según la metodología 
FAO: $\mathrm{ETc}=\mathrm{ET}_{0} \times \mathrm{K}_{\mathrm{c}} \times \mathrm{K}_{\mathrm{L}}$, donde $\mathrm{ET}_{0}$ es la evapotranspiración de referencia [6]; $\mathrm{K}_{\mathrm{c}}$, coeficiente de cultivo [7]; $\mathrm{K}_{\mathrm{L}}$, coeficiente corrector [8].

El contenido volumétrico de agua del suelo $(\theta \mathrm{v})$ en el perfil $0-0,8 \mathrm{~m}$ fue monitorizado con un sensor capacitivo (Diviner2000, Entelechy Pty. Ltd., Golden Grove, Australia). Para ello, se instalaron dos tubos de acceso por repetición $(n=6)$ a $23 \mathrm{~cm}$ del emisor central. $\theta \mathrm{v}$ fue medido cada 2 - 7 días en el periodo de 8:00 - 10:00 h UT durante ambos años experimentales.

El estado hídrico de la planta fue monitorizado a través del potencial hídrico de tallo ( $\Psi$ stem), las microvariaciones del diámetro del tronco (VDT) y de la temperatura de copa (Tc). Istem fue medido al mediodía con una cámara de presión (mod. SF-PRES-70, SolFranc Tecnologías, S.L., Spain) cada 3 7 días en 2 hojas por repetición cercanas al tronco y envueltas con plástico y papel de aluminio [2].

VDT se obtuvieron mediante 6 sensores (2 por repetición) de desplazamiento lineal (LVDT, Solartron Metrology, modelo DF $\pm 2,5 \mathrm{~mm}$, Bognor Regis, UK). A partir de las medidas de las fluctuaciones del diámetro del tronco se obtuvieron los siguientes parámetros [9]: diámetro máximo diario del tronco (MXTD), diámetro mínimo diario del tronco (MNTD), máxima contracción diaria (MDS), calculada como la diferencia entre MXTD y MNTD, y tasa de crecimiento diaria del tronco (TGR), determinada como la diferencia entre MXTD de dos días consecutivos.

Tc fue medida al mediodía con una cámara térmica (Flir One, Flir Systems, Wilsonville, OR, USA) en los mismos días que $\Psi$ stem. Dos imágenes fueron tomadas por repetición $(n=6)$ a una distancia de 1,5 m de la copa y en la parte soleada del árbol [10]. La emisividad fue de 0,95 y las fotografías fueron analizadas en el programa informático Flir Tools application (Flir One, Flir Systems, Wilsonville, OR, USA). Para atenuar el impacto de las variables climáticas, se determinaron dos índices térmicos: (i) la diferencia entre la temperatura de la copa y la del aire $(\Delta \mathrm{T})$; y ii) el índice de estrés hídrico del cultivo (CWSI, crop water stress index) [11]:

$\Delta \mathrm{T}=\mathrm{Tc}-\mathrm{Ta}$

CWSI $=\frac{\Delta \mathrm{T}-\Delta \mathrm{T}_{\mathrm{wet}}}{\Delta \mathrm{T}_{\mathrm{dry}}-\Delta \mathrm{T}_{\mathrm{wet}}}$

donde, Tc es la temperatura de la copa; Ta es la temperatura del aire; $\Delta$ Tdry $\Delta$ Twet es la diferencia entre la temperatura de la copa y la del aire cuando el cultivo tiene los estomas completamente cerrados y $\Delta$ Twet cuando están completamente abiertos. $\Delta$ Twet y $\Delta$ Tdry fueron determinadas de acuerdo con las recomendaciones propuestas por Blaya-Ros et al. [12].

Se determinó el coeficiente de variación (CV), la intensidad de señal (IS) y la sensibilidad de los indicadores del estado hídrico. IS de $\Psi$ stem, MDS, Tc fue calculada como la relación entre los valores (V) de los tratamientos deficitarios (RD) y CTL (IS $=\mathrm{V}_{\mathrm{RD}} \cdot \mathrm{V}_{\mathrm{CTL}^{-1}}$ ) y para $\theta \mathrm{v}$ como IS $=\mathrm{V}_{\mathrm{CTL}} \cdot \mathrm{V}_{\mathrm{RD}}{ }^{-1}$. La sensibilidad se determinó mediante las expresiones:

Sensibilidad: $S=\frac{I S}{C V}$ propuesta por Goldhamer y Fereres [9].

Sensibilidad corregida: $S^{*}=\frac{\mathrm{IS}-1}{\mathrm{CV}}$ propuesta por de la Rosa et al. [13].

El análisis de los datos se realizó mediante ANOVA, utilizando el programa informático SPSS Statistics (SPSS Inc., 24.0 Statistical package; Chicago, IL, USA) y la prueba de rango múltiple de Duncan. Las regresiones lineales entre los indicadores se realizaron con Sigmaplot Plus v.12.5 (Systat Software, San Jose, CA, USA). 


\section{Resultados y discusión}

El régimen de riego influyó en el estado hídrico del suelo (Figuras 1b, 1b) y de la planta (Figura 1c, 1d). En el tratamiento CTL, el contenido volumétrico de agua del suelo medio a $25 \mathrm{~cm}$ de profundidad $(\theta \mathrm{v})$ fue de $37,1 \%$ durante ambos años de estudio. Del mismo modo, los árboles de CTL mostraron un

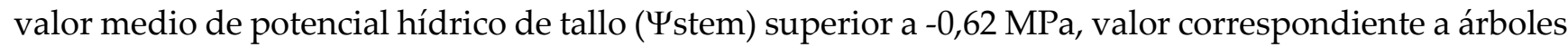
bajo condiciones no limitantes de agua en el suelo [14]. Los árboles de MS y SS presentaron valores de $\theta \mathrm{v}$ y $\Psi$ stem similares a los árboles del tratamiento CTL antes de que el riego fuera restringido. Cuando el riego fue suprimido, $\theta \mathrm{v}$ disminuyó progresivamente a medida que avanzaba el déficit, alcanzando valores mínimos, en el tratamiento MS, de 29,6 \% en 2018 y 29,0 \% en 2019. El tratamiento SS alcanzó un mayor déficit hídrico durante los periodos de supresión, logrando valores mínimos de $27,1 \%$ en 2018 y 26,0 \% en 2019. Los valores de $\Psi$ stem de ambos tratamientos deficitarios disminuyeron de forma gradual hasta alcanzar los umbrales establecidos en cada periodo de supresión del riego. De acuerdo con Blanco et al. [14] y Marsal et al. [15], valores de $\Psi$ stem inferiores a -1,5 MPa pueden repercutir negativamente a la producción y la calidad de la fruta [15].
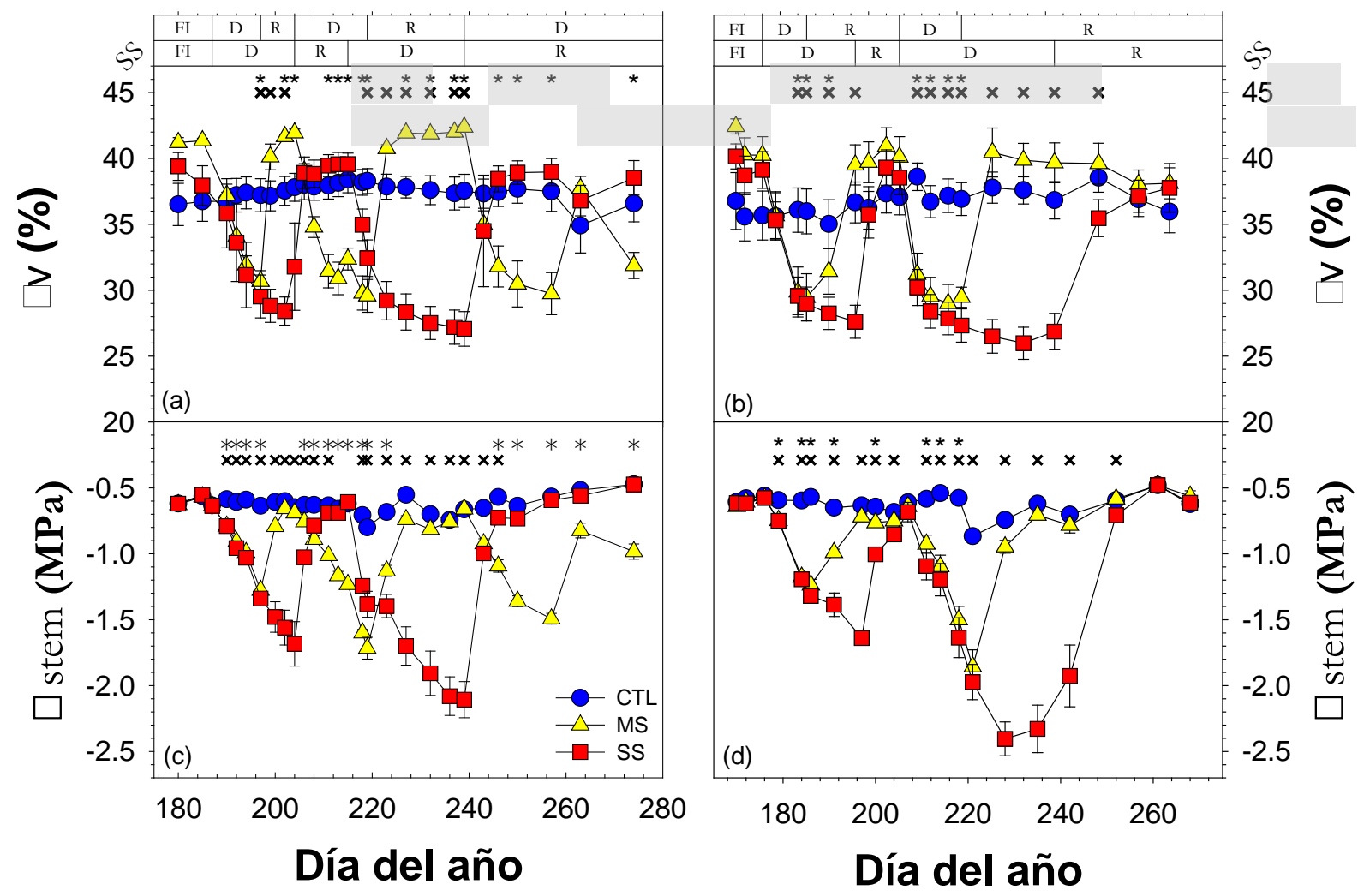

Figura 1. Evolución $(\mathrm{a}, \mathrm{b})$ del contenido volumétrico de agua del suelo a $25 \mathrm{~cm}$ de profundidad $(\theta \mathrm{v})$ y $(\mathrm{c}, \mathrm{d})$ del potencial hí-

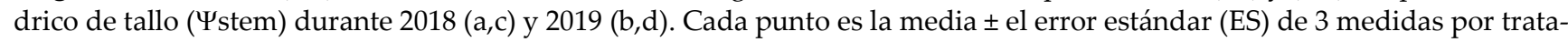
miento para $\theta \mathrm{v}$ y 6 medidas por tratamiento para $\Psi$ stem. Los asteriscos y las cruces indican diferencias significativas entre MS y CTL y entre SS y CTL según el test de Rango Múltiple de Duncan $(p<0,05)$. Las barras horizontales superiores indican los periodos de los ciclos supresión-recuperación del riego que definieron a los tratamientos MS y SS. 'FI' es el periodo previo al inicio de los ciclos de supresión-recuperación, en el que ambos tratamientos deficitarios fueron regados como CTL;

' $\mathrm{D}$ ', periodo de supresión de riego; ' $\mathrm{R}$ ', periodo de recuperación de los árboles. 


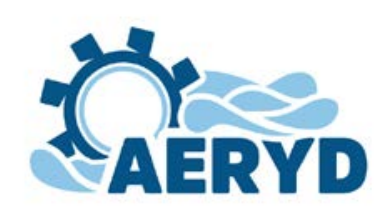
CARTAGENA 2021

Los parámetros derivados de las fluctuaciones del diámetro de tronco, la máxima contracción diaria del tronco (MDS) y la tasa de crecimiento diaria del tronco (TGR), mostraron una rápida respuesta al déficit hídrico (Figura 2). MDS y TGR se vieron fuertemente influenciados por el estrés hídrico. Los tratamientos deficitarios mostraron un aumento de los valores de MDS durante los ciclos de supresión. Por el contrario, los valores de TGR, obtenidos por los tratamientos deficitario, fueron significativamente inferiores a los árboles de CTL. SS alcanzó unos valores máximos de MDS de 235 y $255 \mu \mathrm{m}$ para el primer ciclo de supresión y 400 y $441 \mu$ m para el segundo ciclo de supresión durante 2018 y 2019, respectivamente. Los árboles del tratamiento CTL mostraron unos valores medios de MDS de $96 \mu \mathrm{m}$ en ambos años de experimentación. Los valores de TGR disminuyeron a medida que el ciclo avanzaba, pasando de $290 \mu \mathrm{m} \mathrm{día}^{-1}$, obtenidas a finales de junio, a las $90 \mu \mathrm{m}^{-1}$ obtenidas a finales del estudio, lo que podría indicar una disminución de la elasticidad del tronco [16]. Como se puede observar en la figura $2 a$ y $2 b$, hubo una gran influencia interanual en los valores de MDS, lo que podría ser atribuido al crecimiento del tronco.
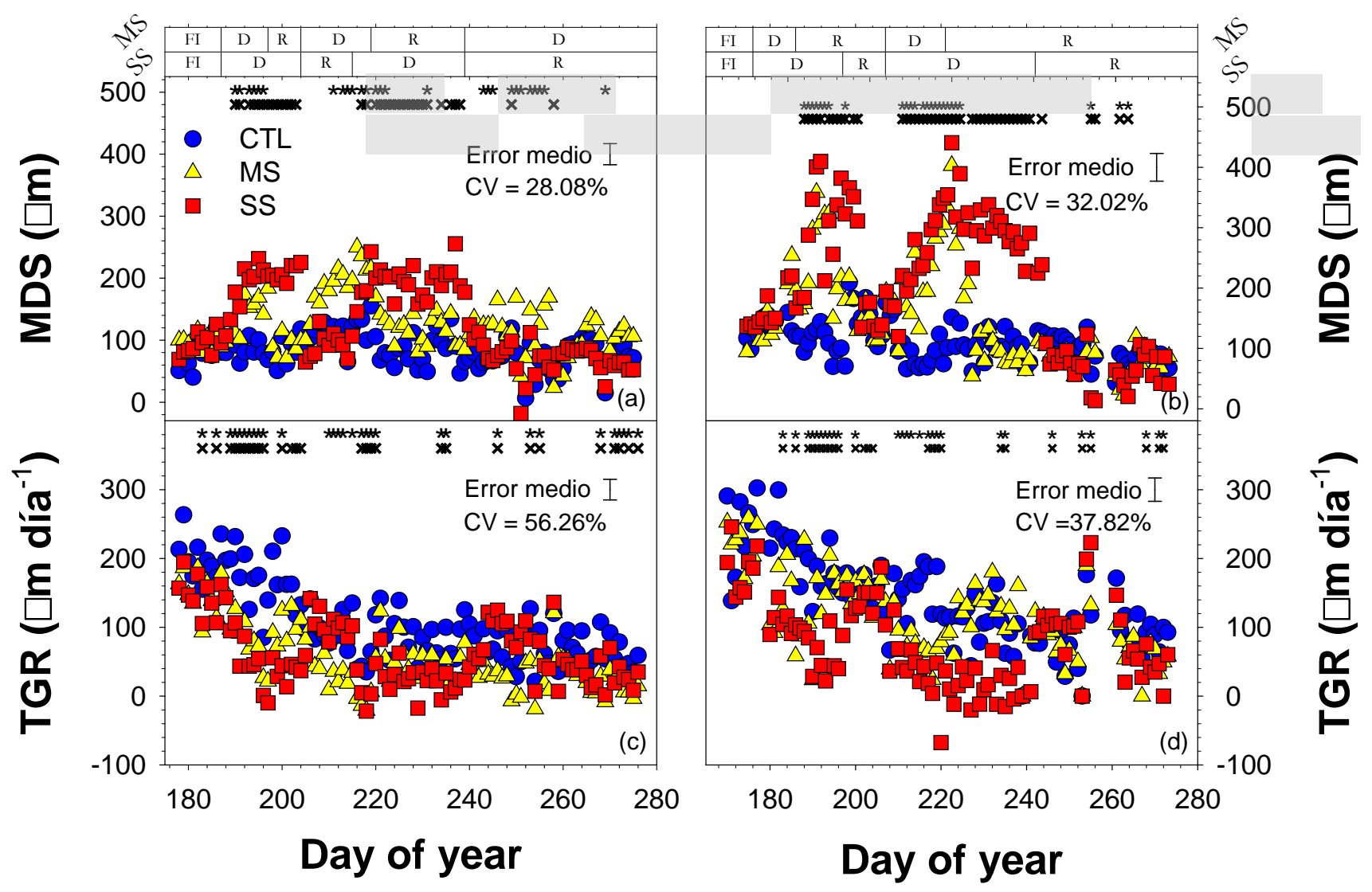

Figura 2. Evolución $(a, b)$ de la máxima contracción diaria del tronco (MDS) y (c,d) de la tasa de crecimiento diaria del tronco (TGR) durante $2018(\mathrm{a}, \mathrm{c})$ y 2019 (b,d). Cada punto es la media \pm ES de 6 medidas por tratamiento. Los asteriscos y las cruces indican diferencias significativas entre MS y CTL y entre SS y CTL según el test de Rango Múltiple de Duncan ( $p<0,05)$. Las barras horizontales superiores indican los periodos de los ciclos supresión-recuperación del riego que definieron a los tratamientos MS y SS. 'FI' es el periodo previo al inicio de los ciclos de supresión-recuperación, en el que ambos tratamientos deficitarios fueron regados como $C T L$; ' $D^{\prime}$, periodo de supresión de riego; ' $R$ ', periodo de recuperación de los árboles. 
Una respuesta similar fue obtenida por Tc y los índices térmicos derivados, $\Delta \mathrm{T}$ y CWSI, al déficit hídrico en 2019 (Figure 3). Los árboles de CTL mostraron una alta tasa transpirativa durante ambos años de estudio, lo que dio lugar a que Tc fuera inferior a Ta $\left(\Delta \mathrm{T}\right.$ media $=-0,77^{\circ} \mathrm{C}$; Figura $\left.3 \mathrm{~b}\right)$. Aunque los árboles CTL mostraron una alta tasa de transpiración, ésta no fue capaz de mantener los valores de CWSI cercanos a 0, máxima apertura estomática. Esto podría indicar que, aunque el agua en el suelo no fue un factor limitante, la resistencia al flujo del agua aumentó [10]. Por el contrario, los tratamientos deficitarios experimentaron un incremento significativo de los valores de Tc (Figura 3a), motivado por una disminución de la disponibilidad hídrica del suelo (Figura 1a, 1b). Este aumento de temperatura posiblemente fue consecuencia de una menor disipación del calor a través de la evaporación del agua [17]. Del mismo modo, los índices térmicos, $\Delta \mathrm{T}$ y CWSI, presentaron valores significativamente superiores a los mostrados por los árboles de CTL (Figura 2c). Los valores de $\Delta \mathrm{T}$ alcanzados por los árboles de SS estuvieron cercanos al límite superior establecido por Blaya-Ros et al. [12], confirmando la intensa regulación estomática. El estrés hídrico también se vio reflejado en los valores de CWSI (Figura 2c), provocando un aumento de los valores de CWSI hasta alcanzar valores próximos a 0,5 y 0,9 durante el primer y el segundo periodo de sequía.
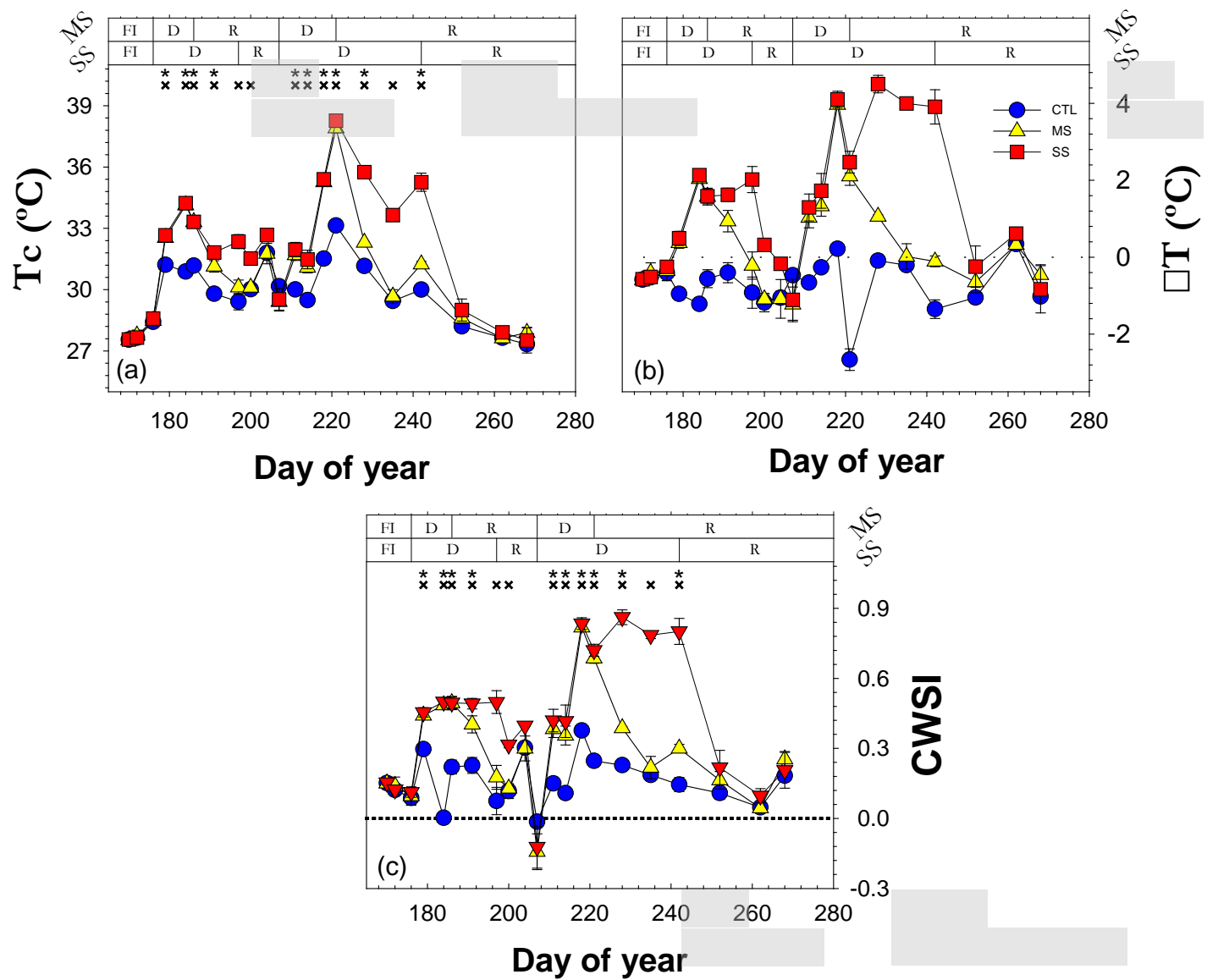

Figura 3. Evolución (a) de la temperatura de la copa (Tc), (b) de la diferencia de temperatura copa-aire (DT) y (c) del índice de estrés del cultivo (CWSI) durante 2019. Cada punto es la media \pm ES de 6 medidas por tratamiento. Los asteriscos y las cruces indican diferencias significativas entre MS y CTL y entre SS y CTL según el test de Rango Múltiple de Duncan (p< 0,05). Las barras horizontales superiores indican los periodos de los ciclos supresión-recuperación del riego que definieron a los tratamientos MS y SS. 'FI' es el periodo previo al inicio de los ciclos de supresión-recuperación, en el que ambos tratamientos deficitarios fueron regados como $C T L$; ' $\mathrm{D}^{\prime}$, periodo de supresión de riego; ' $\mathrm{R}$ ', periodo de recuperación de los árbo- 
La tabla 1 muestra una alta relación entre $\Psi$ stem y el estado hídrico del suelo, determinado por $\theta \mathrm{v}$, lo que indicaría que $\Psi$ stem se ve fuertemente influenciado por la reserva hídrica del suelo. Del mismo modo, Istem mostró una estrecha relación con MDS; sin embargo, esta relación tuvo dos partes claramente diferenciadas: i) una fase lineal para valores de $\Psi$ stem entre $-0,5 \mathrm{MPa}$ y $-1,3 \mathrm{MPa}$, en que una reducción en $\Psi$ stem implica un incremento de MDS; y ii) fase suavemente curvilínea para valores Istem inferiores a -1,3 MPa, donde se da una consolidación de los valores de MDS. En cerezo 'Prime Giant', Blanco et al. [14] observaron que en valores de $\Psi$ stem próximos a -1,3 MPa se producía un agotamiento importante de las reservas hídricas de la planta, y, por lo tanto, de su capacidad de contracción diaria. TGR fue el indicador que menor relación mostró con $\Psi$ stem, resultados similares a los obtenidos por Moriana et al. [18] en olivo, donde observaron que $\Psi$ stem obtenía una mayor relación con MDS que con TGR. Tc y los índices térmicos derivados, $\Delta \mathrm{T}$ y CWSI, obtuvieron un coeficiente de correlación de Pearson superior a 0,8 con $\Psi$ stem, lo que estaría en concordancia con los datos obtenidos en olivo por García-Tejero et al. [19].

Table 1. Coeficiente de correlación de Pearson para las relaciones lineales entre las distintas variables medidas durante el periodo experimental.

\begin{tabular}{l|ccccccc}
\hline & Istem & CWSI & $\Delta \mathbf{T}$ & Tc & MDS & TGR & $\theta \mathbf{v}$ \\
\hline CWSI & $-0,87$ & 1,00 & - & - & - & - & - \\
$\Delta \mathbf{T}$ & -0.88 & 0.85 & 1.00 & - & - & - & - \\
Tc & -0.81 & 0.85 & 0.71 & 1.00 & - & - & - \\
MDS & -0.78 & 0.81 & 0.73 & 0.72 & 1.00 & - & - \\
TGR & 0.56 & -0.46 & -0.51 & -0.47 & -0.40 & 1.00 & - \\
$\theta \mathbf{v}$ & 0.83 & -0.75 & -0.81 & -0.66 & -0.72 & 0.51 & 1.00 \\
\hline
\end{tabular}

El análisis de los distintos indicadores reveló que MDS y $\Psi$ stem tuvieron la mayor sensibilidad durante ambos años de estudio (Figure 4). Aunque MDS mostró unos valores ligeramente superiores a

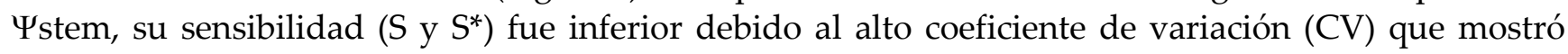
durante el experimento. Blanco et al. [14] observó una respuesta de MDS idéntica en cerezos adultos, donde MDS y $\Psi$ stem obtuvieron unos valores de IS similares; sin embargo, la sensibilidad de MDS fue inferior debido a la alta variación del indicador. Tc y $\theta \mathrm{v}$ mostraron una baja intensidad de señal ante el déficit hídrico, lo que dificultaría su uso para la elaboración de programas de riego utilizando IS. No obstante, ambos indicadores mostraron una alta sensibilidad (S) debido a que registraron un CV bajo, posiblemente debido al rango de medida de estos de ambos indicadores y la su variación dentro de este. Por el contrario, ambos indicadores, Tc y $\theta v$, mostraron una sensibilidad corregida ( $\left.S^{*}\right)$ [13] inferior a MDS y $\Psi$ stem. 

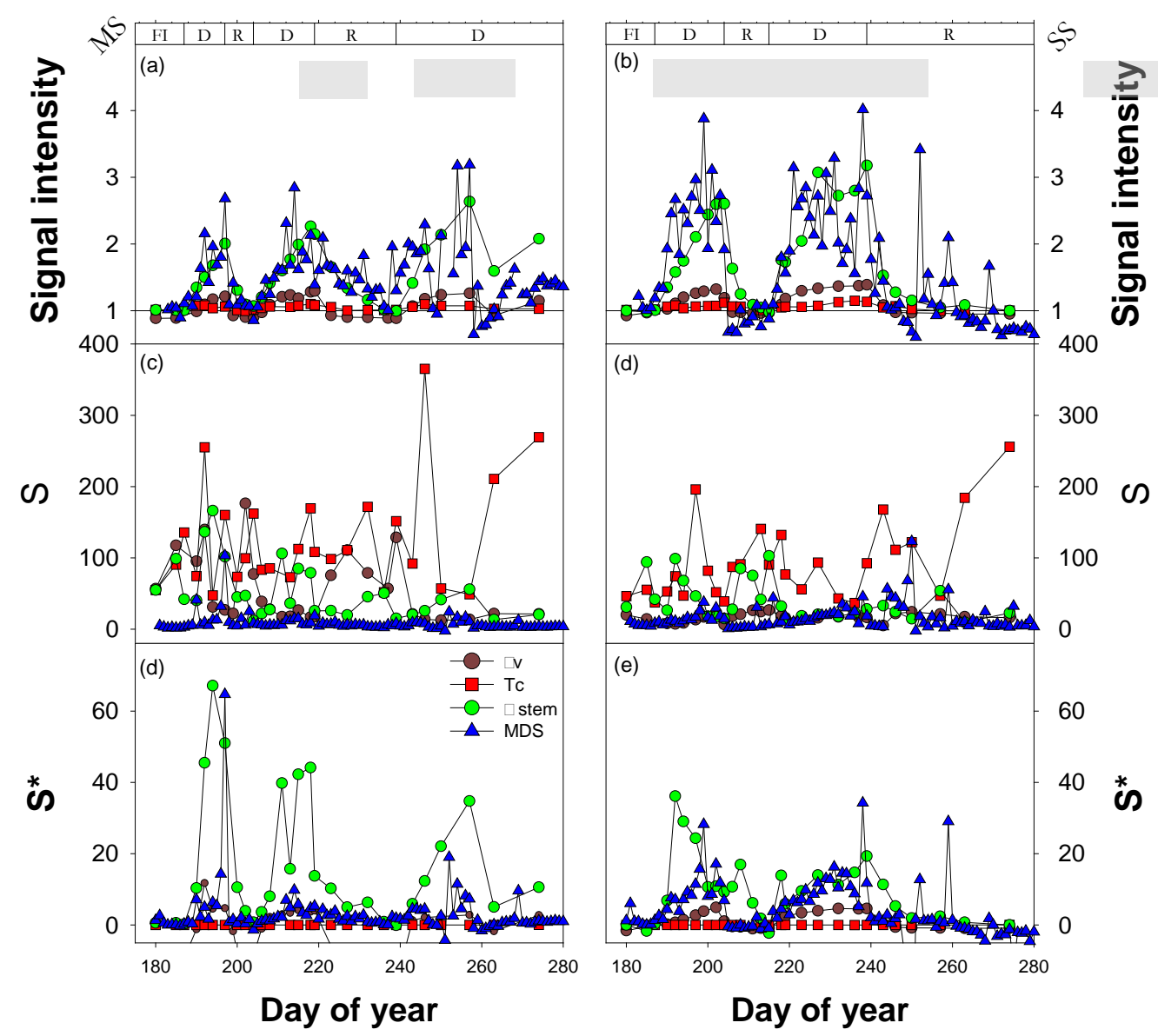

Figura 4. Evolución (a) de la intensidad de señal, (b) de la sensibilidad (S) y (c) de la sensibilidad corregida (S*) durante 2018. Las barras horizontales superiores indican los periodos de los ciclos supresión-recuperación del riego que definieron a los tratamientos MS y SS. 'FI' es el periodo previo al inicio de los ciclos de supresión-recuperación, en el que ambos tratamientos deficitarios fueron regados como CTL; ' $\mathrm{D}$ ', periodo de supresión de riego; ' $\mathrm{R}$ ', periodo de recuperación de los árboles.

\section{Conclusiones}

Los resultados expuestos evidencian que MDS y $\Psi$ stem consiguieron la mayor intensidad de señal durante ambos años de estudio. Sin embargo, la sensibilidad ( $S$ y $S^{*}$ ) de MDS se vio afectada por su alto $\mathrm{CV}$, por lo que haría falta disponer de un mayor número de sensores por parcela para aumentar la precisión de este indicador. La sensibilidad calculada a partir de las dos expresiones (S y $\left.S^{*} ;[9,13]\right)$ para $\theta \mathrm{v}$ y Tc fue contradictoria, obteniendo el método tradicional $(S)$ valores superiores a los registrados por el método corregido $\left(\mathrm{S}^{*}\right)$. Por lo cual, la expresión corregida $\left(\mathrm{S}^{*}\right)$ sería más adecuada para calcular la sensibilidad de los indicadores de estrés hídrico, dado que tiene en cuenta IS y CV.

Todos los indicadores e índices estudiados, a excepción de TGR, podrían usarse para estimar Istem con precisión y evaluar el nivel de estrés al que está sometido el cultivo. MDS podría utilizarse para elaborar programas de riego en niveles de estrés ligero-moderado, desaconsejando su uso en niveles de estrés moderados-severos ( $\Psi$ stem $<-1,3 \mathrm{MPa}$ ). $\Delta \mathrm{T}$ y CWSI mostraron una estrecha relación con Istem; sin embargo, no sería recomendado su uso para la confección de programas de riego mediante el método de la intensidad de señal. No obstante, podrían emplearse para estudiar y evaluar la respuesta de cerezos jóvenes al déficit hídrico y, por lo tanto, para supervisar programas de riego basados en niveles de estrés diferentes. 


\section{Referencias}

1. Jones, H. G. Monitoring plant and soil water status: established and novel methods revisited and their relevance to studies of drought tolerance. J. Exp. Bot. 2007, 58, 119-130.

2. Schackel, K. A.; Ahmadi, H.; Biasi, W.; Burchner, R.; Goldhamer, D. A.; Gurusinghe, S.; Hasey, J.; Kester, D.; Krueger, B.; Lampinen, B.; et al. Plant water status as an index for irrigation need in deciduous fruit trees. Hort Technol. 1996, 7, $23-29$.

3. Puerto, P.; Domingo, R.; Torres, R.; Pérez-Pastor, A.; García-Riquelme, M. Remote management of deficit irrigation in almond trees based on maximum daily trunk shrinkage. Water relations and yield. Agric. Water Manag. 2013, 126, 33-45.

4. Ortuño, M. F.; Conejero, W.; Moreno, F.; Moriana, A.; Intrigliolo, D. S.; Biel, C.; Mellisho, C. D.; Pérez-Pastor, A.; Domingo, R.; Ruiz-Sánchez, M. C.; et al. Could trunk diameter sensors be used in woody crops for irrigation scheduling? A review of current knowledge and future perspectives. Agric. Water Manag. 2010, 97, 1-11.

5. García-Tejero, I. F.; Gutiérrez-Gordillo, S.; Ortega-Arévalo, C.; Iglesias-Contreras, M.; Moreno, J. M.; Souza-Ferreira, L.; Durán-Zuazo, V. H. Thermal imaging to monitor the crop-water status in almonds by using the non-water stress baselines. Sci. Hortic. 2018, 238, 91-97.

6. Allen, R.G.; Pereira, L.S.; Raes, D.; Smith, M. FAO Irrigation and drainage paper In Crop. Evapotranspiration-Guidelines for Computing Crop. Water Requirements; Food and Agriculture Organization: Rome, Italy, 1998.

7. Marsal, J. FAO irrigation and drainage paper In Crop. Yield Response Water. Sweet Cherry; Food and Agriculture Organization: Rome, Italy, 2012; pp. 449-457

8. Fereres, E.; Pruitt, W.O.; Beutel, J.A.; Henderson, D.W.; Holzapfel, E.; Schulbach, H.; Uriu, K. Evapotranspiration and drip irrigation scheduling. In Drip Irrigation Management; Leaflet 21259; Division of Agricultural Sciences, University of California: Los Angeles, CA, USA, 1981; pp. 8-13.

9. Goldhamer, D. A.; Fereres, E. Irrigation scheduling protocols using continuously recorded trunk diameter measurements. Irrig. Sci. 2001, 20, 115-125.

10. Jackson, R. D.; Kustas, W. P.; Choudhury, B. J. A reexamination of the crop water stress index. Irrig. Sci. 1988, 9, $309-317$.

11. Jackson, R.D.; Idso, S.J.; Reginato, R.J.; Pinter, P.J. Canopy temperature as a crop water stress indicator. Water Resour. Res. 1981, 17, 1133-1138.

12. Blaya-Ros, P. J.; Blanco, V.; Domingo, R.; Soto-Valles, F.; Torres-Sánchez, R. Feasibility of low-cost thermal imaging for monitoring water stress in young and mature sweet cherry trees. Appl. Sci. 2020, 10.

13. de la Rosa, J. M.; Conesa, M. R.; Domingo, R.; Pérez-Pastor, A. A new approach to ascertain the sensitivity to water stress of different plant water indicators in extra-early nectarine trees. Sci. Hortic. 2014, 169, 147-153.

14. Blanco, V.; Domingo, R.; Pérez-Pastor, A.; Blaya-Ros, P. J.; Torres-Sánchez, R. Soil and plant water indicators for deficit irrigation management of field-grown sweet cherry trees. Agric. Water Manag. 2018, 208, 83-94.

15. Marsal, J.; Lopez, G.; del Campo, J.; Mata, M.; Arbones, A.; Girona, J. Postharvest regulated deficit irrigation in 'Summit' sweet cherry: fruit yield and quality in the following season. Irrig. Sci. 2010, 28, 181-189.

16. Conesa, M. R.; Torres, R.; Domingo, R.; Navarro, H.; Soto, F.; Pérez-Pastor, A. Maximum daily trunk shrinkage and stem water potential reference equations for irrigation scheduling in table grapes. Agric. Water Manag. 2016, 172, 51-61.

17. Jones, H. G. Use of infrared thermometry for estimation of stomatal conductance as a possible aid to irrigation scheduling. Agric. For. Meteorol. 1999, 95, 139-149.

18. Moriana, A.; Girón, I. F.; Martín-Palomo, M. J.; Conejero, W.; Ortuño, M. F.; Torrecillas, A.; Moreno, F. New approach for olive trees irrigation scheduling using trunk diameter sensors. Agric. Water Manag. 2010, 97, 1822-1828.

19. García-Tejero, I. F.; Hernández, A.; Padilla-Díaz, C. M.; Diaz-Espejo, A.; Fernández, J. E. Assessing plant water status in a hedgerow olive orchard from thermography at plant level. Agric. Water Manag. 2017, 188, 50-60. 